\title{
A Raman study of ion irradiated icy mixtures
}

\author{
G. Ferini, G. A. Baratta, and M. E. Palumbo \\ INAF, Osservatorio Astrofisico di Catania, Via S. Sofia 78, 95123 Catania, Italy \\ Received 19 May 2003 / Accepted 25 September 2003

\begin{abstract}
In this paper we present a Raman study of pure $\mathrm{CH}_{4}, \mathrm{H}_{2} \mathrm{O}: \mathrm{CH}_{4}: \mathrm{N}_{2}$ and $\mathrm{CH}_{3} \mathrm{OH}: \mathrm{N}_{2}$ frozen films before and after ion irradiation at $12 \mathrm{~K}, 100 \mathrm{~K}$ and $300 \mathrm{~K}$. By means of Raman spectroscopy, we monitor the structural evolution of each film, whose chemical and physical properties are deeply modified by the interaction with the ion beam. For the two methane containing samples, Raman spectra show that the initial ice is partially converted into a refractory residue, which under further irradiation evolves towards an amorphous carbon (AC) with a band near $1560 \mathrm{~cm}^{-1}$ (G line) and a shoulder at about $1360 \mathrm{~cm}^{-1}$ (D line). No evidence of the AC Raman band is seen in the spectra of the methanol-containing mixture. By means of Lorentzian fits, we have determined the specific parameters of the AC band ( $G$ and D line peak positions, widths and relative intensities) in our spectra after ion irradiation and we have compared them with the corresponding parameters of the band as observed in the spectra of 11 IDPs (Interplanetary Dust Particles). Here we present the experimental results and discuss their contribution to our knowledge of the origin and evolution of IDPs.
\end{abstract}

Key words. astrochemistry - molecular processes - methods: laboratory - techniques: spectroscopic comets: general - interplanetary medium

\section{Introduction}

Comets are believed to be among the less altered objects in the Solar System (and hence the best reservoir of presolar material) and one of the main sources of interplanetary dust grains that are collected in our atmosphere and analysed in terrestrial laboratories. It is known that the external layers of comets are exposed to background radiation (galactic cosmic rays) that, according to some models, can drive the formation of an organic crust, which can survive when the comet approaches the Sun (e.g., Strazzulla \& Johnson 1991). Raman spectroscopy is one of the laboratory techniques used to get information about the constituent materials of these grains. Furthermore it has been used in the laboratory to study the effects of ion irradiation on the formation and further evolution of refractory carbonaceous materials. By means of in situ Raman spectroscopy, it has been shown that amorphous carbon can be formed at low temperatures after ion irradiation of butane and benzene (Strazzulla \& Baratta 1992). On the basis of these results it has been suggested that an organic crust could already be formed during ion irradiation at low temperature, i.e. while comets stay in the Oort cloud ( $T \sim 10-20 \mathrm{~K}$ ), without requiring a first passage by the Sun and its consequent heating. Once a comet is formed, the inner layers are somehow shielded, and are altered only by the most energetic but less abundant ions. The frozen gases in these regions can sublimate when the comet approaches the

Send offprint requests to: M. E. Palumbo,

e-mail: mepalumbo@ct.astro.it
Sun, passing through the porous structure of the crust. In fact, experiments on targets much thicker than the penetration depth of irradiating ions showed that an organic residue is left over after the sublimation of underlying ices, thus giving support to the hypothesis that an ion-produced cometary organic crust can "survive" ejection from deeper layers (i.e. passages near the Sun) and be as old as the comet itself (Strazzulla et al. 1991). Thus, a dark surface can be formed, which could exhibit strong albedo variations corresponding to patches or fissures in the crust through which volatiles are expelled. Albedo variations have indeed been confirmed by recent observations (Soderblom et al. 2002) of the comet 19P/Borrelly, whose nucleus has an average geometric albedo of only $0.03 \pm 0.005$, with darker spots ranging down to $\sim 0.01$.

Interplanetary dust particles (IDPs) are believed to derive mainly from comets and asteroids (a fraction of them has an interstellar origin), and they are the smallest and most finegrained extraterrestrial objects available for laboratory investigation. Recent studies (e.g., Joswiak et al. 2000; Thomas et al. 1993) have underlined that morphological and mineralogical differences exist between asteroidal and cometary IDPs. Morphologically, asteroidal IDPs tend toward smoother, compact forms while cometary IDPs exhibit fluffy, porous, aggregate textures. Mineralogically, asteroidal IDPs are often composed of phyllosilicate minerals with only minor amounts of anhydrous phases present, while cometary IDPs are dominated by anhydrous phases and may contain significant carbon and GEMS. The average density obtained for asteroidal 
IDPs is $2.5 \mathrm{~g} \mathrm{~cm}^{-3}$ whereas cometary IDP densities average $1.1 \mathrm{~g} \mathrm{~cm}^{-3}$. However, it can be difficult to unambiguously classify an individual IDP as either asteroidal or cometary since characteristics of each group can be found within the other. The presence of carbon in IDPs was first detected by Fraundorf et al. (1982) and confirmed by subsequent Raman studies (McKeegan et al. 1984; Fahey et al. 1985; Macklin et al. 1987; Wopenka 1988; Raynal et al. 2001). The amount of carbon can be greatly variable (Thomas et al. 1995); anyway it has been found that the $\mathrm{C}$-rich material is amorphous or poorly crystalline and could present vescicular textures (Keller et al. 1994; Thomas et al. 1993). The IDPs' amorphous carbon (AC) could be the result of ion irradiation processes of volatile materials (frozen hydrocarbons in the parent bodies) and thus it is interesting to compare the profile of the AC band in such IDPs with that of ion-irradiated laboratory samples.

In this paper we present Raman spectra of three icy samples (pure $\mathrm{CH}_{4}$ and the mixtures $\mathrm{H}_{2} \mathrm{O}: \mathrm{CH}_{4}: \mathrm{N}_{2}$ and $\mathrm{CH}_{3} \mathrm{OH}: \mathrm{N}_{2}$ ) irradiated with $\mathrm{He}^{+}(30 \mathrm{keV}), \mathrm{Ar}^{+}(30 \mathrm{keV})$ or $\mathrm{Ar}^{++}(60 \mathrm{keV})$ ions at low temperature $(12 \mathrm{~K})$ and then gradually warmed up. Previous experiments performed on pure frozen hydrocarbons, such as benzene and butane (Strazzulla \& Baratta 1992) have shown that ion irradiation at low doses makes the samples fluorescent and, at higher doses, a broad band appears around $1560 \mathrm{~cm}^{-1}$, which is attributed to amorphous carbon. A detailed analysis of this feature makes it possible to infer information on the structural properties of a given sample. The new experiments here discussed show that ion irradiation can drive the formation of amorphous carbon also in a pure methane ice or in hydrocarbon-bearing mixtures (e.g. $\mathrm{H}_{2} \mathrm{O}: \mathrm{CH}_{4}: \mathrm{N}_{2}$ ). Amorphous carbon samples formed after ion irradiation of icy mixtures will be referred to as IPHAC (Ion Produced Hydrogenated Amorphous Carbon). Moreover, we compare the AC band of laboratory samples to that of 11 IDPs. These IDPs are some out of the 20 that Wopenka (1988) analyzed by means of Raman spectroscopy (in particular we considered the ones in whose spectra the AC Raman feature has a higher signal-to-noise ratio). This analysis showed that these particles can be divided in six different groups depending on the profile of the amorphous carbon feature. Particles which exhibit an amorphous carbon feature that indicates a high degree of order have been assigned to group 1 while those with a decreasing degree of order have been classified in groups 2 to 5. Particles which do not show the amorphous carbon feature have been assigned to group 6. Among laboratory samples we have considered icy mixtures after ion irradiation (IPHAC) here discussed and hydrogenated amorphous carbon grains (HAC) produced from non-ice experiments (e.g., Mennella et al. 2001, 2002). We have found that the characteristics of the AC band observed in the spectra of the three icy mixtures discussed in this paper differ from those exhibited by IDPs, implying that the corresponding structural properties are different while the amorphous carbon band profile of HAC is similar to that of IDPs.

In the following section we will briefly describe the experimental set-up, then we will give some general information on the profile of the amorphous carbon band and on what can be inferred from it. The results of the experiments performed and the Raman spectra of the films are presented in Sect. 4. The astrophysical implications are discussed in Sect. 5.

\section{Experimental set-up}

The in situ analyses have been performed in a stainless steel vacuum chamber facing a Raman spectrometer. Figure 1 schematically shows the arrangement used for in situ Raman spectroscopy. A continuous Ar-ion laser beam $(\lambda=514 \mathrm{~nm})$ enters a confocal illuminator perpendicular to its optical axis, into which it is deflected by a microprism. The confocal optical system is arranged in such a way that any parallel beam incident along the optical axis in the opposite direction of the laser is focused onto the entrance slit of the spectrometer. By means of two flat mirrors, the laser beam is reflected towards the vacuum chamber and focused by an objective into a $40 \mu \mathrm{m}$ spot on the sample. The back-scattered Raman light is collimated by the same objective into a parallel beam that, according to the setup geometry, goes back along the same path of the laser beam and reaches the confocal illuminator where it is focused onto the entrance slit of the spectrometer.

Inside the vacuum chamber, in which the pressure is kept below $10^{-7}$ mbar, a substrate (crystalline silicon) is placed in thermal contact with a cold finger whose temperature can be varied between $10 \mathrm{~K}$ and $300 \mathrm{~K}$. The vacuum chamber is interfaced with an ion implanter ( $30 \mathrm{kV}$; Danfysik) from which ions with energy up to $30 \mathrm{keV}$ (60 keV for double ionizations) can be obtained. The ion beam produces a $2 \times 2 \mathrm{~cm}^{2}$ spot on the target and currents in the range of $100 \mathrm{nA} \mathrm{cm}^{-2}$ to a few $\mu \mathrm{A} \mathrm{cm}^{-2}$.

A needle valve is used to admit pre-prepared gases (or mixtures) into the chamber, where they freeze on the substrate. A He-Ne laser can be used to monitor the thickness of the ice film during accretion; this is achieved by looking at the interference pattern (intensity versus time) given by the laser beam reflected at an angle of $45^{\circ}$ both by the vacuum-film and film-substrate interfaces (see Baratta \& Palumbo 1998 for further details on the technique used to measure the thickness).

The substrate holder is mounted at an angle of $45^{\circ}$ both with the ion beam and the laser beam. This configuration offers the advantage that spectra can be easily taken in situ, even during irradiation, without tilting the sample.

The amount of energy released into the sample (dose) is expressed in units of $\mathrm{eV}$ per small molecule $(16 \mathrm{amu})$ and is calculated from the knowledge of the ion fluence $F$ (ions $\mathrm{cm}^{-2}$ ), the stopping power $S\left(\mathrm{eV} \mathrm{cm}{ }^{2}\right.$ molecule $\left.{ }^{-1}\right)$ for the chosen projectile or its penetration depth (range) $r$ into the target (molecules $\mathrm{cm}^{-2}$ ). The first is given by a current integrator on the path of the ion beam, which measures the charge that reaches the sample during irradiation; the other two parameters are easily calculated by using available routines (e.g. TRIM, etc.). Thus, if the penetration depth is greater than the sample thickness, the dose is:

$d=S \times F$.

If the range is smaller than the sample thickness, the ions are stopped in the target and only the molecules within a certain depth in the ice, roughly equal to the range, interact with the 


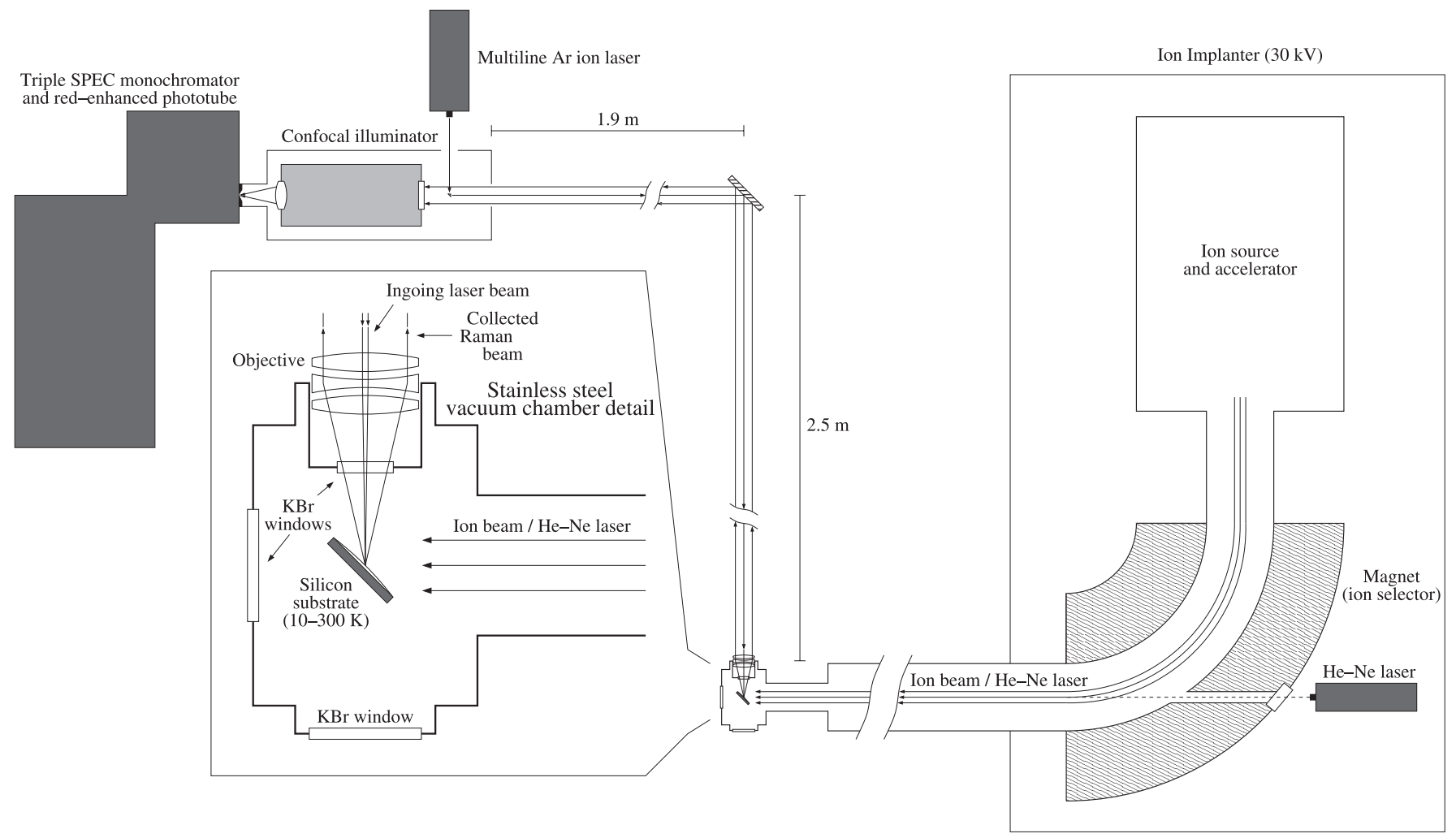

Fig. 1. Schematic view of the experimental apparatus used for in situ Raman spectroscopy of ion irradiated frozen gases.

ion beam. In this case the average energy absorbed by each molecule is:

$d=r^{-1} \times F \times E$,

where $E$ is the projectile energy given in $\mathrm{eV}$.

The icy films we report on in the following are all several $\mu \mathrm{m}$ thick, while the penetration depth of the ions used as projectiles ranges between $0.05 \mu \mathrm{m}$ and $0.5 \mu \mathrm{m}$. This implies that the incoming ions stop in the target and only the outer layers of the ice are processed.

\section{Raman spectroscopy of carbonaceous materials}

Raman spectroscopy gives valuable information on the vibrational transitions of molecules in the solid, liquid, and gas phase. In particular it is a powerful tool to investigate the structural properties of a given sample and so it has often been used to study the effects of ion-induced lattice damage in carbonaceous solids and organic compounds (Elman et al. 1981; Strazzulla \& Baratta 1992; Baratta et al. 1996; Musumeci et al. 2000; Strazzulla et al. 2001 and references therein). Raman spectroscopy makes it possible to perform mineralogical studies (see for example Wang et al. 2000), to identify substances and chemical compounds, and to deduce structural and symmetry properties of molecules. For these abilities Raman analysis may have important applications in planetology, especially in the "in situ" and/or laboratory studies concerned with the evolution of surface rocks and also in the determination of past environments, as recorded by meteorites. Interplanetary dust grains can also be analysed by Raman spectroscopy, inferring information on the composition and processing suffered by their parent bodies.

In this paper we will concentrate on what can be deduced from the study of the $1560 \mathrm{~cm}^{-1} \mathrm{AC}$ band. The profile of this band differs from one carbonaceous material to another, reflecting the great variation in chemical composition and structure ("degree of order"), and this makes Raman spectroscopy a powerful technique of classification for samples which exhibit this characteristic feature.

Generally speaking, Raman spectroscopists associate the crystallite size to the crystal order or "perfection". In HOPG (Highly Ordered Pyrolitic Graphite) or natural graphite with large $(>1 \mu \mathrm{m})$ microcrystals a band is present at about $1582 \mathrm{~cm}^{-1}$ (Robertson 1986), which is called the $\mathrm{G}$ (or graphitic) line. Because of the momentum conservation selection rule, this is the only active first-order strong Raman band and it is due to the $\mathrm{E}_{2 g}$ mode (Tuinstra \& Koenig 1970). The spectra of microcrystalline $(\leq 1 \mu \mathrm{m})$ graphite show an additional line peaked at $1350 \mathrm{~cm}^{-1}$, called the $\mathrm{D}$ (or disorder) line.

It has been found (Tuinstra \& Koenig 1970) that a correlation exists between the intensity ratio $I_{\mathrm{D}} / I_{\mathrm{G}}$ and the coherence length $L_{a}$ of the crystal, which can be taken as an indicator of the degree of order: $I_{\mathrm{D}} / I_{\mathrm{G}} \propto 1 / L_{a}$ if $2.5 \mathrm{~nm}<$ $L_{a}<1 \mu \mathrm{m}$. In more disordered carbons $\left(L_{a}<2.5 \mathrm{~nm}\right)$ this correlation is no longer valid; for example, in amorphous carbons with a low degree of order an opposite trend is observed. In this case, the $I_{\mathrm{D}} / I_{\mathrm{G}}$ ratio increases with the cluster size. An indication of the degree of order can be also provided by the $\mathrm{G}$ and $\mathrm{D}$ bands width: the broader the bands are, the more 
disordered the amorphous carbon is. In fact, since the FWHM of both $\mathrm{G}$ and $\mathrm{D}$ lines depends on the bond angle disorder, and on the relative amounts of crystallites versus the amorphous matrix, a broadening of the bands indicates an increase in the bond angle disorder and a decrease in the mean size and/or number of crystallites. Below a certain size of the basic structural units of the carbonaceous material, the bands become so wide that they can no longer be recognized as individual peaks (Wopenka 1988).

Carbonaceous materials with different degree of order differ in number and/or size of the $\mathrm{sp}^{2}$ clusters. When the sample is ion-irradiated, these parameters change. Several laboratory experiments performed on carbonaceous materials have shown that ion irradiation can increase or decrease the $\mathrm{sp}^{2}$ cluster size depending on the starting material. During ion irradiation two competitive processes are present: graphitization and amorphization. The first one is related to the energy release in the form of heat ("thermal spikes") inside the collision cascade $\left(\mathrm{sp}^{3}\right.$ to $\mathrm{sp}^{2}$ conversion with or without hydrogen loss), while the second is caused by displacement collisions and depends on the size of crystallites. Since the relaxation time of the excitation generated by an ion is of the order of one picosecond or less, equilibrium processes (like annealing) are not possible. The saturation effect is due to the competition between these two processes (Compagnini et al. 1992), in which saturation has been reached when they become equal.

The effects of releasing energy to a poorly ordered amorphous carbon by thermal annealing are different. Experiments have been performed on AC samples with a low degree of order (Dillon et al. 1984; Vidano \& Fishbach 1981; Vidano 1980), showing that the $I_{\mathrm{D}} / I_{\mathrm{G}}$ ratio increases up to a maximum value at $T=800-900^{\circ} \mathrm{C}$ and then it goes to zero at higher annealing temperature. In summary, energetic processing greatly affects $G$ and $D$ lines peak positions, widths and relative intensities, because these parameters are directly linked to the lattice structure, which changes after ion irradiation and/or annealing. In order to qualitatively analyse these variations and particularly the relative contribution of the D and G lines, we have done a Lorentzian line shape fitting analysis for the amorphous carbon band in the Raman spectra of the laboratory samples. A similar analysis was performed for the band observed in the spectra of some IDPs.

\section{Results}

We present the results obtained for three different icy mixtures containing hydrocarbons: pure $\mathrm{CH}_{4}, \mathrm{H}_{2} \mathrm{O}: \mathrm{CH}_{4}: \mathrm{N}_{2}$ (1:6:3) and $\mathrm{CH}_{3} \mathrm{OH}: \mathrm{N}_{2}$ (1:2). Each mixture was first deposited at $12 \mathrm{~K}$, then irradiated (with non-reactive ions such as $\mathrm{He}^{+}$or $\mathrm{Ar}^{++}$) and finally warmed gradually to room temperature. Raman spectra of the samples were taken for different doses. In Figs. 2, 3 and 7 the corresponding infrared spectra of the mixtures as deposited (top curves) are also shown for comparison. Since a molecule can have infrared active transitions which are not Raman-active (or extremely weak) and vice versa (because of the different selection rules), some features present in the IR spectrum are absent from the Raman spectrum. For example, water bands, very intense in the IR spectrum of thin icy

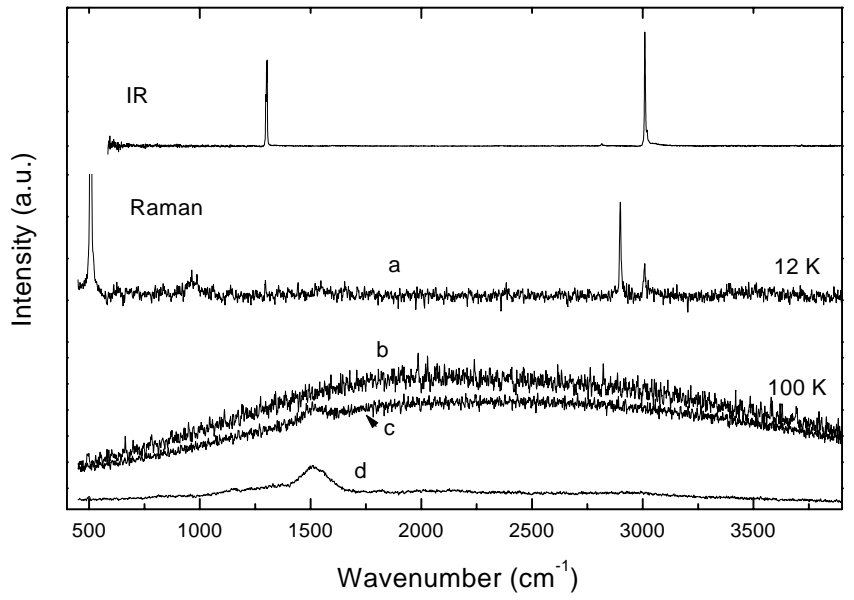

Fig. 2. IR (top curve) and Raman spectra of a pure methane frozen film before (curve a) and after (curves b-d) irradiation with $\mathrm{He}^{+}(30 \mathrm{keV})$ and $\mathrm{Ar}^{++}(60 \mathrm{keV})$ ions. Spectrum a (corresponding to the mixture as deposited) has been arbitrarily shifted with respect to the other Raman spectra for clarity. Curves b, c and d represent the spectra, at $100 \mathrm{~K}$, after increasing irradiation doses $(130,290$ and $1000 \mathrm{eV} / 16 \mathrm{amu}$ respectively).

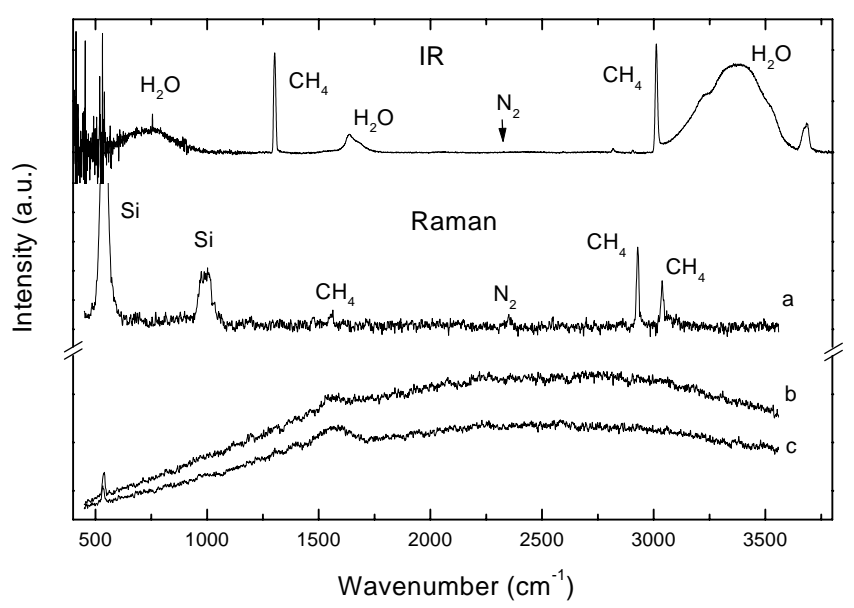

Fig. 3. IR (top curve) and Raman spectra of the mixture $\mathrm{H}_{2} \mathrm{O}: \mathrm{CH}_{4}: \mathrm{N}_{2}$ before (curve a) and after (curves b and c, corresponding to doses of $378 \mathrm{eV} / 16 \mathrm{amu}$ and $825 \mathrm{eV} / 16 \mathrm{amu}$ respectively) irradiation with $\mathrm{He}^{+}(30 \mathrm{keV})$ ions at low temperature $(12 \mathrm{~K})$. In spectra $\mathrm{b}$ and $\mathrm{c}$ the ice features are undetectable because of the intense fluorescence background. The arrow in the IR spectrum indicates the position of the $\mathrm{N} \equiv \mathrm{N}$ symmetric stretching mode, which cannot be observed.

films, cannot be easily observed in the Raman spectrum (because of the small cross-section), while the opposite is the case for the symmetric modes of mono- and poly-nuclear molecules such as $\mathrm{N}_{2}$ and $\mathrm{CH}_{4}$ (which are IR-inactive). These considerations are summarized in Table 1 (where we also provide the observed peak positions of the Raman features in our spectra) and indicate that the two spectroscopic techniques can be used as complementary tools in identifying the solid-phase features of various molecules. 
Table 1. IR and Raman features of the molecules studied here.

\begin{tabular}{|c|c|c|c|c|c|}
\hline \multirow[t]{2}{*}{ Molecule } & \multirow[t]{2}{*}{ Vibrational mode } & \multicolumn{2}{|c|}{ Position $\left(\mathrm{cm}^{-1}\right)$} & \multirow[t]{2}{*}{ IR } & \multirow[t]{2}{*}{ Raman } \\
\hline & & IR & Raman & & \\
\hline $\mathrm{H}_{2} \mathrm{O}$ & OH sym $\&$ asym stretch & 3300 & $3400^{*}$ & active & active \\
\hline $\mathrm{H}_{2} \mathrm{O}$ & $\mathrm{OH}$ bend & 1660 & $1640^{*}$ & active & active \\
\hline $\mathrm{H}_{2} \mathrm{O}$ & libration & 675 & 800 & active & active \\
\hline $\mathrm{CH}_{4}$ & C-H stretch (asym) & 3010 & 3011 & active & active \\
\hline $\mathrm{CH}_{4}$ & C-H stretch (sym) & & 2900 & inactive & active \\
\hline $\mathrm{CH}_{4}$ & C-H deform (sym) & 1534 & 1547 & inactive & active \\
\hline $\mathrm{CH}_{4}$ & C-H deformation & 1300 & & active & inactive \\
\hline $\mathrm{CH}_{3} \mathrm{OH}$ & O-H stretch & 3260 & $3360^{*}$ & active & active \\
\hline $\mathrm{CH}_{3} \mathrm{OH}$ & C-H stretch (asym) & 2961 & 2950 & active & active \\
\hline $\mathrm{CH}_{3} \mathrm{OH}$ & C-H stretch (sym) & 2830 & 2829 & active & active \\
\hline $\mathrm{CH}_{3} \mathrm{OH}$ & bend \& def & 1460 & 1470 & active & active \\
\hline $\mathrm{CH}_{3} \mathrm{OH}$ & bend \& def & 1126 & 1107 & active & active \\
\hline $\mathrm{CH}_{3} \mathrm{OH}$ & C-O stretch & 1034 & 1021 & active & active \\
\hline $\mathrm{CH}_{3} \mathrm{OH}$ & torsion & 694 & & active & inactive \\
\hline $\mathrm{N}_{2}$ & $\mathrm{~N} \equiv \mathrm{N}$ strecth & 2328 & 2322 & inactive & active \\
\hline
\end{tabular}

* Liquid phase position.

Table 2. List of studied icy mixtures.

\begin{tabular}{clcccccc}
\hline \hline Number & Sample & Ratio & $\begin{array}{c}\text { Thickness } \\
(\mu \mathrm{m})\end{array}$ & Ion \& energy & $\begin{array}{c}\text { Fluence } \\
\left(\text { ions cm }{ }^{-2}\right)\end{array}$ & $\begin{array}{c}\text { Total dose } \\
(\mathrm{eV} / 16 \mathrm{amu})\end{array}$ & $\begin{array}{c}\text { Temperature } \\
(\mathrm{K})\end{array}$ \\
\hline 1 & $\mathrm{CH}_{4}$ & & 3.7 & $\mathrm{He}^{+}, 30 \mathrm{keV}$ & $1.8 \times 10^{15}$ & 64 & 12 \\
& & & & $\mathrm{He}^{+}, 30 \mathrm{keV}$ & $1.8 \times 10^{15}$ & 130 & 100 \\
& & & & $\mathrm{Ar}^{++}, 60 \mathrm{keV}$ & $1.1 \times 10^{15}$ & 1000 & 100 \\
2 & $\mathrm{H}_{2} \mathrm{O}: \mathrm{CH}_{4}: \mathrm{N}_{2}$ & $1: 6: 3$ & 5 & $\mathrm{He}^{+}, 30 \mathrm{keV}$ & $3.2 \times 10^{16}$ & 825 & 12 \\
& & & & $\mathrm{He}^{+}, 30 \mathrm{keV}$ & $2.0 \times 10^{15}$ & & 300 \\
3 & $\mathrm{CH}_{3} \mathrm{OH}: \mathrm{N}_{2}$ & $1: 2$ & 10 & $\mathrm{He}^{+}, 30 \mathrm{keV}$ & $1.2 \times 10^{16}$ & 164 & 12 \\
& $\mathrm{CH}_{3} \mathrm{OH}: \mathrm{N}_{2}$ & $1: 2$ & 10 & $\mathrm{Ar}^{++}, 60 \mathrm{keV}$ & $1.8 \times 10^{15}$ & 654 & 12 \\
& & & & $\mathrm{Ar}^{+}, 30 \mathrm{keV}$ & $7.1 \times 10^{16}$ & 1900 & 12 \\
\hline
\end{tabular}

\subsection{Irradiation effects}

It is well known that when fast ions impinge on an icy sample, many molecular bonds are broken, and fragments are formed along the ion track which recombine giving rise to molecular species not present in the original sample. Beyond the chemical modifications, ion irradiation drives a gradual alteration of the sample structure, causing a rearrangement of the atoms in their lattice sites and a thorough modification of the optical properties of the ice. As we will show, Raman spectroscopy is a powerful tool for monitoring structural changes. In the next sections we will illustrate the effects of ion irradiation on the Raman spectra of the mixtures reported in Table 2. When different ions have been used to irradiate the same sample, we report in the table the maximum fluence of each ion and the total dose suffered after each irradiation step.

\subsection{Pure methane ice}

Figure 2 shows the evolution of the Raman spectrum of a pure methane frozen film after irradiation with $30 \mathrm{keV} \mathrm{He}^{+}$ and $60 \mathrm{keV} \mathrm{Ar}^{++}$ions. The curve labelled with the letter $a$ represents the spectrum of the sample as deposited at $12 \mathrm{~K}$, in which the features due to silicon (substrate) phonon vibrations $\left(\sim 525 \mathrm{~cm}^{-1}\right.$ and $\sim 990 \mathrm{~cm}^{-1}$ for the first and the second order, respectively) are clearly distinguishable. In addition, we see the $\mathrm{C}-\mathrm{H}$ asymmetric and symmetric stretching modes and the $\mathrm{C}-\mathrm{H}$ symmetric deformation mode of $\mathrm{CH}_{4}$ molecules, that peak around $3010,2900 \mathrm{~cm}^{-1}$ and $1550 \mathrm{~cm}^{-1}$ respectively. After irradiation at $12 \mathrm{~K}$ with $30 \mathrm{keV} \mathrm{He}^{+}$ions (up to a dose of $64 \mathrm{eV} / 16 \mathrm{amu}$ ), the sample was warmed up to $100 \mathrm{~K}$ (spectrum not shown), a temperature at which underlying unirradiated $\mathrm{CH}_{4}$ had already sublimated, and then further 
irradiated with different ions $\left(30 \mathrm{keV} \mathrm{He} \mathrm{He}^{+}, 60 \mathrm{keV} \mathrm{Ar}^{++}\right)$up to $1000 \mathrm{eV} / 16$ amu. From infrared spectroscopy (e.g., Kaiser \& Roessler 1998; Baratta et al. 2002; Moore \& Hudson 2003) we know that ion irradiation of pure methane produces other hydrocarbons (such as ethane, $\mathrm{C}_{2} \mathrm{H}_{6}$, and propane, $\mathrm{C}_{3} \mathrm{H}_{8}$ ), which sublimate during warm up at about $60-80 \mathrm{~K}$, and a refractory residue stable at room temperature (e.g., Strazzulla et al. 1983; Strazzulla \& Johnson 1991). The formation of an organic residue is a general ion irradiation effect and occurs for different icy mixtures, as has been discussed elsewhere (Moore et al. 1983; Foti et al. 1984; Strazzulla \& Johnson 1991). The nature of the residue formed and its thermal stability depend on several parameters such as the initial composition of the ice and the irradiation dose. The "organic residue" obtained by ion irradiation of frozen icy mixtures containing $\mathrm{C}, \mathrm{H}, \mathrm{N}$, and $\mathrm{O}$ bearing species is mainly constituted by $\mathrm{C}=\mathrm{C}, \mathrm{C}=\mathrm{O}, \mathrm{C} \equiv \mathrm{N}, \mathrm{C}-\mathrm{H}$ and $\mathrm{O}-\mathrm{H}$ bonds, as shown by infrared spectroscopy analysis (Strazzulla et al. 2001).

In Fig. 2 the curves labelled with $b, c$ and $d$ show the spectra of the residue for increasing irradiation doses at $100 \mathrm{~K}$ : 130, 290 and $1000 \mathrm{eV} / 16$ amu. In these spectra the silicon features are no longer detectable, implying that the icy film optical properties have been modified (darkening) so that the laser beam is absorbed in the sample and can no longer reach the substrate. Furthermore, after irradiation, an intense fluorescence continuum appears, typical of disordered polymerlike carbons containing a relatively large quantity of hydrogen, and due to radiative recombination of electron-hole pairs produced by laser irradiation. With increasing irradiation fluence, the fluorescence signal becomes weaker and weaker, due to the gradual loss of hydrogen. When higher doses are reached, a broad band appears near $1560 \mathrm{~cm}^{-1}$, which is attributed to $\mathrm{C}=\mathrm{C}$ stretching vibration of amorphous carbon. These effects were already known for other simple frozen hydrocarbons, both aromatic such as benzene $\left(\mathrm{C}_{6} \mathrm{H}_{6}\right)$ and aliphatic such as butane $\left(\mathrm{C}_{4} \mathrm{H}_{10}\right.$; Strazzulla \& Baratta 1992$)$. In summary ion irradiation of frozen methane induces the following effects:

- at low fluences, the sample shows an intense fluorescence signal due to the formation of a damaged molecular solid;

- as irradiation goes on, hydrogen is lost and the fluorescence signal intensity decreases; the Raman feature of amorphous carbon appears and gets more and more intense.

All these effects occur at low temperature and cannot be investigated using infrared spectroscopy, which testifies to the chemical evolution of the sample, but gives little information on its structure.

A fit of the profile of the amorphous carbon feature after the highest dose of irradiation with two Lorentzian components gives $v_{\mathrm{G}}=1517 \mathrm{~cm}^{-1}, F W H M_{\mathrm{G}}=146 \mathrm{~cm}^{-1}, \nu_{\mathrm{D}}=1265 \mathrm{~cm}^{-1}$, $F W H M_{\mathrm{D}}=314 \mathrm{~cm}^{-1}$, and $I_{\mathrm{D}} / I_{\mathrm{G}}=0.232$.

\subsection{The mixture $\mathrm{H}_{2} \mathrm{O}: \mathrm{CH}_{4}: \mathrm{N}_{2}$}

Figure 3 shows the spectra of an icy mixture $\left(\mathrm{H}_{2} \mathrm{O}: \mathrm{CH}_{4}: \mathrm{N}_{2}\right.$ (1:6:3)) before and after irradiation with $30 \mathrm{keV} \mathrm{He}^{+}$ions at $12 \mathrm{~K}$. Nitrogen $\left(\sim 2350 \mathrm{~cm}^{-1}\right.$, for the symmetric stretching

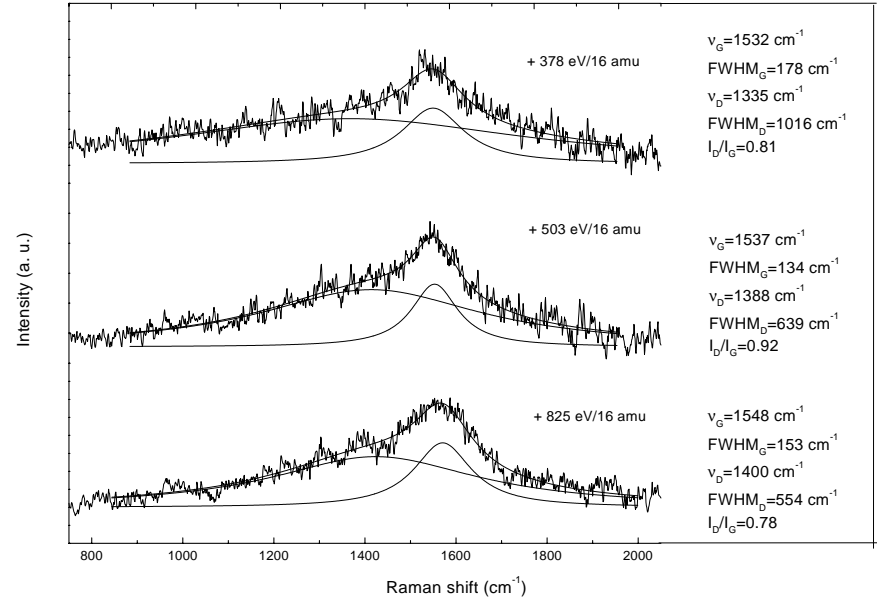

Fig. 4. $\mathrm{G}$ and D Raman bands of the mixture $\mathrm{H}_{2} \mathrm{O}: \mathrm{CH}_{4}: \mathrm{N}_{2}$ after irradiation with $\mathrm{He}^{+}(30 \mathrm{keV})$ ions at $12 \mathrm{~K}$. From top to bottom the spectra are displayed in order of increasing irradiation dose. For each spectrum the Lorentzian fit, with the two components, is shown. The corresponding parameters (peak position, full width at half maximum $(F W H M)$ and the intensity ratio of the $\mathrm{G}$ and D lines) are listed on the right side of the figure.

mode) and methane features are present, while the Raman features of water ice are undetectable.

The amorphous carbon band is distinguishable at doses of $378 \mathrm{eV} / 16 \mathrm{amu}$ (the corresponding spectrum is labelled with letter "b" in the figure) and its profile and intensity vary as irradiation goes on (the spectrum labelled with the letter "c" refers to the sample after a dose of $825 \mathrm{eV} / 16 \mathrm{amu}$ ). Figure 4 illustrates the result of the fitting procedure with Lorentzian components; the corresponding fitting parameters are shown on the right hand side of the figure.

We can see, in particular, that the G-line peak position shifts to higher frequencies with increasing dose. These results are consistent with an increasing size or number of the largest $\mathrm{sp}^{2}$ clusters (with respect to the $\mathrm{sp}^{3}$ clusters) induced by ion irradiation (Baratta et al. 1996).

After irradiation at low temperature of the mixture $\mathrm{H}_{2} \mathrm{O}: \mathrm{CH}_{4}: \mathrm{N}_{2}$, the icy sample was warmed up to room temperature and left under vacuum overnight and then the spectrum was taken (Fig. 5, top curve). Since the penetration depth of the impinging ions was smaller than the sample thickness, just a thin layer of the target was altered by irradiation and so converted into a refractory material. No features due to the ice constituents are obviously present in the spectrum at room temperature, because the underlying ice has sublimated passing through the porous structure of the residue. Only the $\mathrm{C}=\mathrm{C} 1560 \mathrm{~cm}^{-1}$ band is observed superimposed on the fluorescence continuum background.

The organic residue has been further irradiated at room temperature with $\mathrm{He}^{+}(30 \mathrm{keV})$ ions. As we can see from Fig. 5, during irradiation the fluorescence background intensity decreases, as occurs at low temperature.

If we look at the fitting parameters reported in Fig. 6, we note that the $I_{\mathrm{D}} / I_{\mathrm{G}}$ ratio decreases (from 0.74 to 0.57 ) during irradiation. Moreover, the $F W H M$ of both G and D lines 


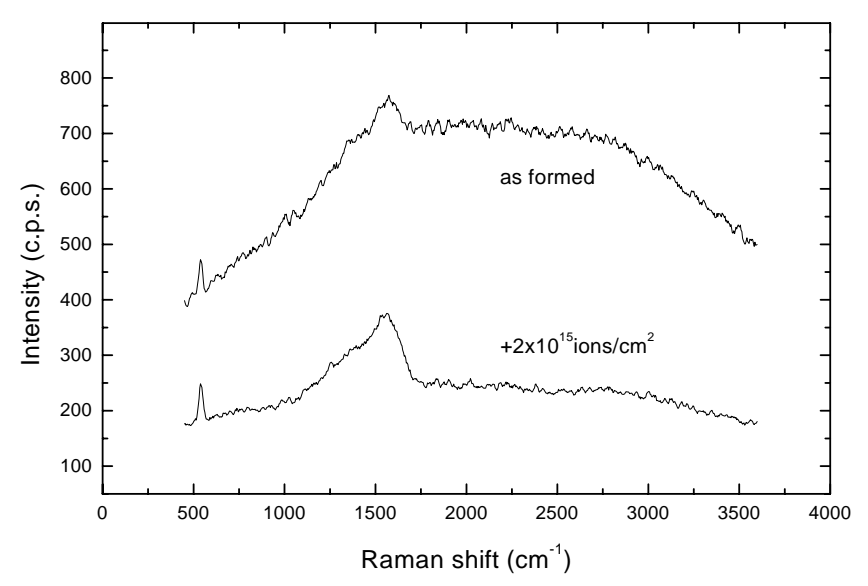

Fig. 5. Raman spectra, at room temperature, of the residue obtained after ion irradiation of the mixture $\mathrm{H}_{2} \mathrm{O}: \mathrm{CH}_{4}: \mathrm{N}_{2}$ at $12 \mathrm{~K}$ before (top curve) and after (bottom curve) irradiation with $30 \mathrm{keV} \mathrm{He}^{+}$ions. It is evident that the fluorescence background greatly decreases after irradiation.

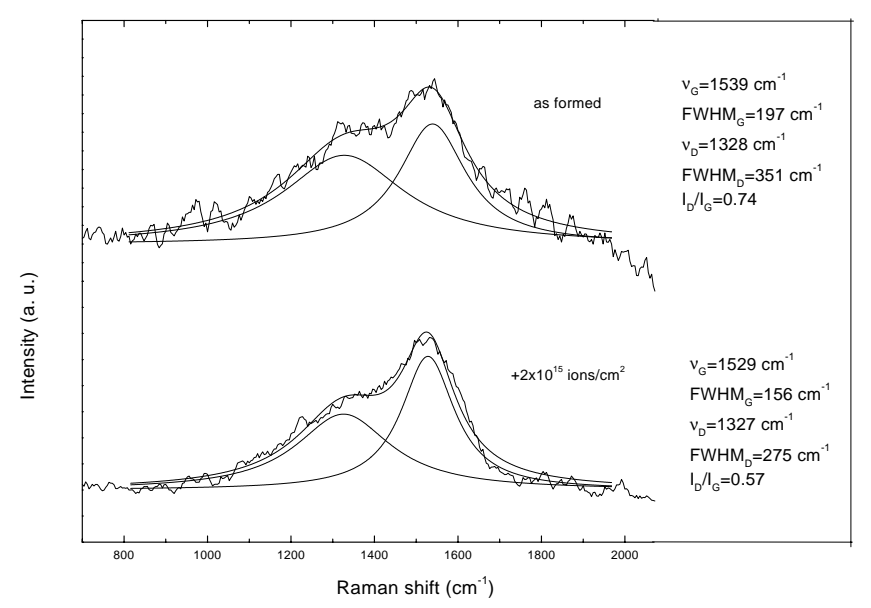

Fig. 6. $G$ and $D$ Raman bands of the residue of the mixture $\mathrm{H}_{2} \mathrm{O}: \mathrm{CH}_{4}: \mathrm{N}_{2}$ before (top curve) and after (bottom curve) irradiation with $\mathrm{He}^{+}(30 \mathrm{keV})$ ions at room temperature. For each spectrum the Lorentzian fit, with the two components, is shown. The corresponding parameters (peak position, Full Width at Half Maximum ( $F W H M$ ) and the intensity ratio of the $\mathrm{G}$ and $\mathrm{D}$ lines) are listed on the right hand side of the figure.

decreases and the $\mathrm{G}$ line peak position shifts from 1539 to $1529 \mathrm{~cm}^{-1}$.

\subsection{The mixture $\mathrm{CH}_{3} \mathrm{OH}: \mathrm{N}_{2}$}

Finally we describe the spectral modifications induced by ion irradiation on a frozen film made up of methanol and nitrogen. The corresponding Raman spectra at $12 \mathrm{~K}$, both before (curve a) and after (curve b) ion irradiation, are presented in Fig. 7. In this case we can see all the original features even after irradiation with a total fluence of $10^{16} \mathrm{He}^{+}$ions $\mathrm{cm}^{-2}$, because of the low fluorescence background. The corresponding dose is nearly $160 \mathrm{eV} / 16 \mathrm{amu}$, and no bands around $1560 \mathrm{~cm}^{-1}$ are observed. Other irradiation experiments with a $\mathrm{CH}_{3} \mathrm{OH}: \mathrm{N}_{2}$ mixture have shown that no features appeared around $1560 \mathrm{~cm}^{-1}$ even after irradiation with $\mathrm{Ar}^{++}$and $\mathrm{Ar}^{+}$up to a maximum

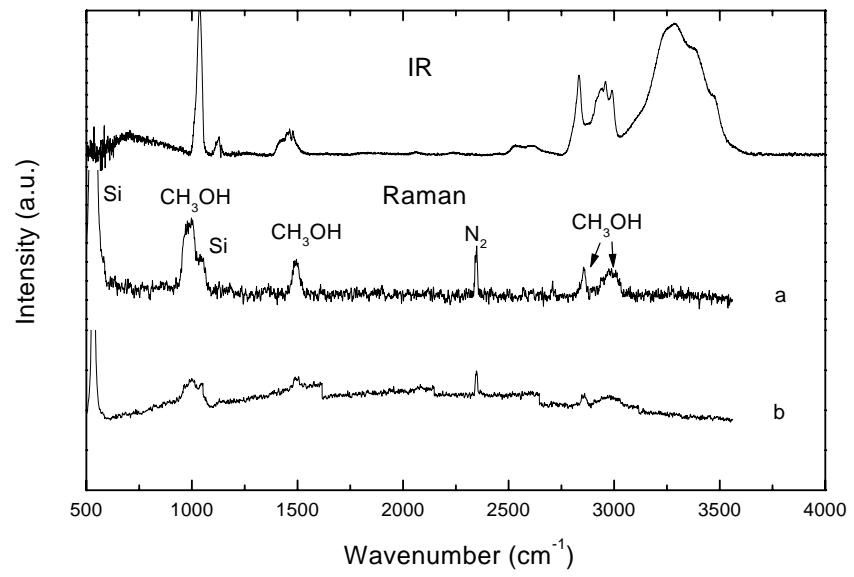

Fig. 7. IR (top curve) and Raman spectra of the mixture $\mathrm{CH}_{3} \mathrm{OH}: \mathrm{N}_{2}$ before (curve a) and after (curve b) irradiation with $\mathrm{He}^{+}(30 \mathrm{keV})$ ions. The fluorescence continuum increases after ion irradiation, but no band appear which can be attributed to the amorphous carbon. The apparent jumps from a spectral region to another indicate laser induced alteration of the sample.

dose of about $1900 \mathrm{eV} / 16 \mathrm{amu}$. On the other hand, we have discussed in the previous sections that the amorphous carbon band is apparent in the spectra of the two methane containing samples at a lower dose. These results indicate that the $\mathrm{AC}$ formation efficiency depends on the specific composition of the ice film and requires an energy release that can vary over a wide range. Further experiments are necessary to see if IPHAC can be effectively formed irradiating icy mixtures containing methanol as carbon bearing species. In the case of a hydrocarbon, such as methane, benzene, etc., doses of some $10-10^{2} \mathrm{eV} / \mathrm{mol}$ are sufficient to make the $1560 \mathrm{~cm}^{-1}$ band observable.

\section{Discussion}

In this paper we have discussed Raman spectra of three ionirradiated icy mixtures at low $(12 \mathrm{~K}, 100 \mathrm{~K})$ and at room temperature. Raman spectroscopy is useful to understand the progressive evolution of the sample towards an amorphous carbon. The characteristic feature of such a disordered structure is the $1560 \mathrm{~cm}^{-1}$ band, which forms at low temperature. It was already kwown that amorphous carbon (IPHAC) can be formed after ion irradiation of icy samples of pure hydrocarbons such as $\mathrm{C}_{4} \mathrm{H}_{10}$ and $\mathrm{C}_{6} \mathrm{H}_{6}$ (both aliphatic and aromatic). The new experiments here discussed show that the formation of IPHAC occurs not only for pure hydrocarbons $\left(\mathrm{CH}_{4}\right)$, but also in the case of a mixture containing them. This supports the idea that the process of IPHAC formation is general. Anyway, there seem to be great differences in the efficiency of the process itself (Sect. 4.4).

The typical parameters (peak position, width and intensity of both $\mathrm{G}$ and $\mathrm{D}$ lines) of the $\mathrm{AC}$ band depend on the carbonaceous material considered. In Figs. 8 and 9 we compare the $F W H M$ of $\mathrm{G}$ and $\mathrm{D}$ lines, versus the corresponding peak position, for different carbonaceous material, which are: $a$ ) IPHAC (samples 1 and 2 of Table 2) both at low and room temperature, $b$ ) different kinds of hydrogenated carbon 
Table 3. Parameters of Lorentzian fit to the Raman spectra of 11 IDPs. Particle names have been assigned by Wopenka (1988).

\begin{tabular}{lccccc}
\hline \hline Particle & $\begin{array}{c}\text { G line position } \\
\left(\mathrm{cm}^{-1}\right)\end{array}$ & $\begin{array}{c}\text { G line } F W H M \\
\left(\mathrm{~cm}^{-1}\right)\end{array}$ & $\begin{array}{c}\text { D line position } \\
\left(\mathrm{cm}^{-1}\right)\end{array}$ & $\begin{array}{c}\text { D line } F W H M \\
\left(\mathrm{~cm}^{-1}\right)\end{array}$ & $I_{\mathrm{D}} / I_{\mathrm{G}}$ \\
\hline Tyson & 1594 & 73 & 1350 & 141 & 1.068 \\
Attila & 1594 & 72 & 1345 & 202 & 0.936 \\
Essex & 1591 & 82 & 1361 & 267 & 0.896 \\
Summersville & 1589 & 100 & 1393 & 469 & 1.392 \\
Elias & 1579 & 101 & 1347 & 288 & 1.326 \\
Lea & 1584 & 159 & 1379 & 269 & 0.978 \\
Mosquito & 1562 & 114.3 & 1349 & 476 & 1.044 \\
Pattonsburg & 1577 & 118 & 1373 & 373 & 1.129 \\
SkyFB & 1591 & 90 & 1371 & 159 & 0.663 \\
Spray & 1581 & 126 & 1364 & 338 & 0.872 \\
Verona & 1585 & 104 & 1358 & 284 & 0.966 \\
\hline
\end{tabular}

Table 4. Parameters of Lorentzian fit to the Raman spectra of amorphous carbon samples from non-ice experiments (Baratta et al. 1996; Mennella et al. 2003).

\begin{tabular}{lcccccc}
\hline \hline Sample & $\begin{array}{c}\text { Fluence } \\
\left(30 \mathrm{keV} \mathrm{He}^{+} \mathrm{cm}^{-2}\right)\end{array}$ & $\begin{array}{c}\text { G line position } \\
\left(\mathrm{cm}^{-1}\right)\end{array}$ & $\begin{array}{c}\mathrm{G} \text { line } F W H M \\
\left(\mathrm{~cm}^{-1}\right)\end{array}$ & $\begin{array}{c}\text { D line position } \\
\left(\mathrm{cm}^{-1}\right)\end{array}$ & $\begin{array}{c}\text { D line } F W H M \\
\left(\mathrm{~cm}^{-1}\right)\end{array}$ & $I_{\mathrm{D}} / I_{\mathrm{G}}$ \\
\hline ACAR & & 1589 & 86 & 1372 & 355 & 1.037 \\
ACAR_H & & 1585 & 102 & 1360 & 284 & 0.953 \\
ACAR_H & & 1585 & 114 & 1363 & 307 & 1.032 \\
ACAR_H & $9.5 \times 10^{15}$ & 1553 & 159 & 1356 & 463 & 0.679 \\
ACH2 & $6.0 \times 10^{15}$ & 1551 & 147 & 1356 & 388 & 0.667 \\
ACH2 & $1.1 \times 10^{16}$ & 1557 & 162 & 1363 & 408 & 0.716 \\
\hline
\end{tabular}

grains (labelled ACH2, ACAR, and ACAR_H ${ }^{1}$ ) produced in the laboratory from non-ice experiments and $c$ ) some IDPs. The mentioned laboratory materials (organic residues obtained after ion irradiation of frozen gases and mixtures, hydrogenated carbon grains) have been considered to test if different kinds of AC samples could be similar, at least from a structural point of view, to the carbon phase of interplanetary grains. Here we have selected 11 different IDPs among the 20 already studied by Wopenka (1988), choosing the ones whose Raman spectra have a higher signal-to-noise ratio. These particles, whose dimensions are between 1 and $50 \mu \mathrm{m}$, have been collected by NASA spacecrafts at altitudes between 8 and $20 \mathrm{~km}$ and have been studied by means of microRaman spectroscopy, infrared transmission spectroscopy, scanning electron microscopy (SEM) and energy-dispersive X-ray

\footnotetext{
$1 \mathrm{ACH} 2$ was prepared by condensation of carbon vapor produced by an arc discharge between two carbon rods in a 10 mbar hydrogen atmosphere (Mennella et al. 2001). The label ACAR refers to hydrogenfree carbon grains produced by laser ablation of carbon electrodes in an argon atmosphere. In some cases, these grains were hydrogenated (ACAR_H) by exposure to hydrogen atoms produced by microwaveexcited dissociation of molecular hydrogen (further details on the preparation of these grains can be found in Mennella et al. 2002). Some of these samples have been irradiated with $30 \mathrm{keV} \mathrm{He}{ }^{+}$ions (Baratta et al. 1996; Mennella et al. 2003).
}

(EDX) analyses (e.g., Sandford \& Walker 1985). It has been found that the particles are heterogeneous, with different mineralogical and structural properties. They contain silicates, in variable amounts, (whose Raman features have not been observed probably due to the presence of carbonaceous material that masks the signal of silicates), and amorphous carbon with different degree of order, as can be seen from Table 3, in which we report the fitting parameters derived by the Lorentzian fits to the IDPs' AC band. The fitting parameters derived by the Lorentzian fits of the AC band for the samples obtained from non-ice experiments are reported in Table 4. In the case of ACAR_H samples, two different specimens were analysed and, as expected, the fit parameters are very similar.

Here, we compare the "laboratory" and the "interplanetary" values of these parameters and try to test the hypothesis that the amorphous carbon phase of IDPs could be similar to ion irradiated ices. In fact, according to some models (Strazzulla \& Johnson 1991), the surface layers of comets $(0.1-0.5 \mathrm{~m})$ are exposed to an irradiation dose of up to $600 \mathrm{eV} /$ molecule. This energetic processing drives the formation of a substantial "crust" of non-volatile material, left over when volatile material from the deeper layers sublimes in the passages near the Sun. Part of this crust, in the form of IDPs, could enter the Earth atmosphere and then be analysed by means of laboratory techniques. Looking at Fig. 8 and particularly at Fig. 9, that illustrate D 


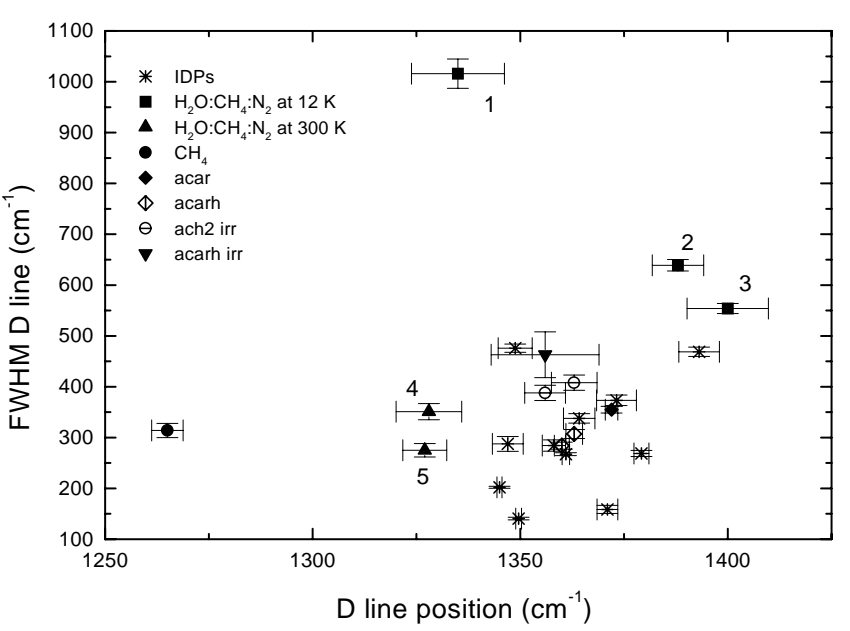

Fig. 8. D line full width at half maximum $(F W H M)$ versus peak position. The stars, all in the middle-bottom part of the figure, refer to IDPs. The label 1 on the squares indicates the mixture $\mathrm{H}_{2} \mathrm{O}: \mathrm{CH}_{4}: \mathrm{N}_{2}$ at $12 \mathrm{~K}$ at the first irradiation dose, while 2 and 3 refer to the same sample at increasing irradiation dose. The same criterion is adopted for the corresponding residue, which is represented by the up triangles and is labelled with 4 and 5 respectively before and after irradiation. Labels ach2 irr and acarh irr refer to ACH2 and ACAR_H grains, respectively, after ion irradiation.

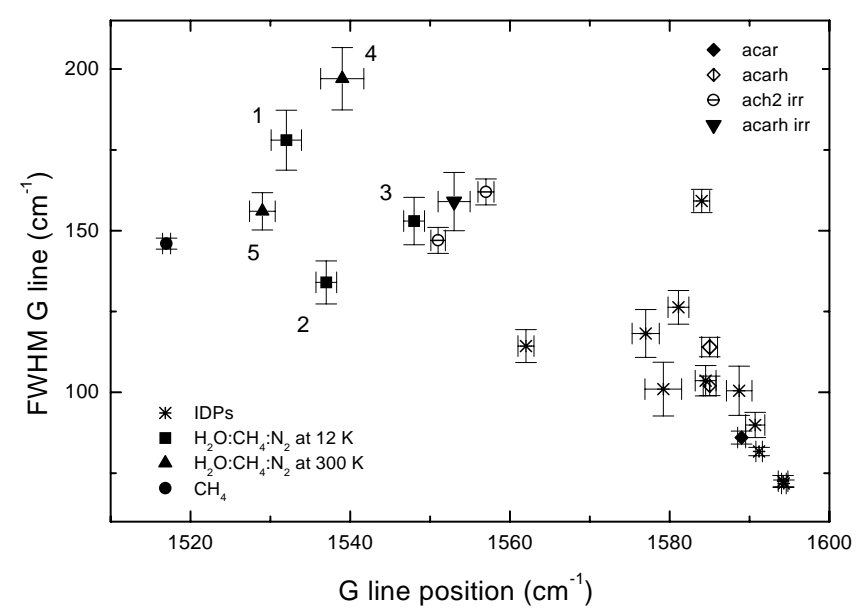

Fig. 9. G line full width at half maximum $(F W H M)$ versus peak position. All the symbols in the graph have the same meaning as in the previous figure.

and $\mathrm{G}$ line widths versus the corresponding peak position, we can say that most of amorphous carbon samples obtained after ion irradiation of icy mixtures greatly differ from IDPs. On the other hand the profiles of the amorphous carbon band in ACAR and ACAR_H are similar to those of IDPs. The latter have a narrower $\mathrm{G}$ line, which peaks at higher wavenumbers than the $\mathrm{AC}$ produced after ion irradiation of icy mixtures, implying that the structure of IDPs is more ordered than that of the IPHACs here considered.

The same difference between laboratory and extraterrestrial samples is apparent in Fig. 10, where the $I_{\mathrm{D}} / I_{\mathrm{G}}$ ratio versus the $\mathrm{G}$ line width is reported. From the figure it is evident that only a few data points, relative to unirradiated ACAR samples, are close to some IDPs, namely Pattonsburg, Mosquito and Verona.

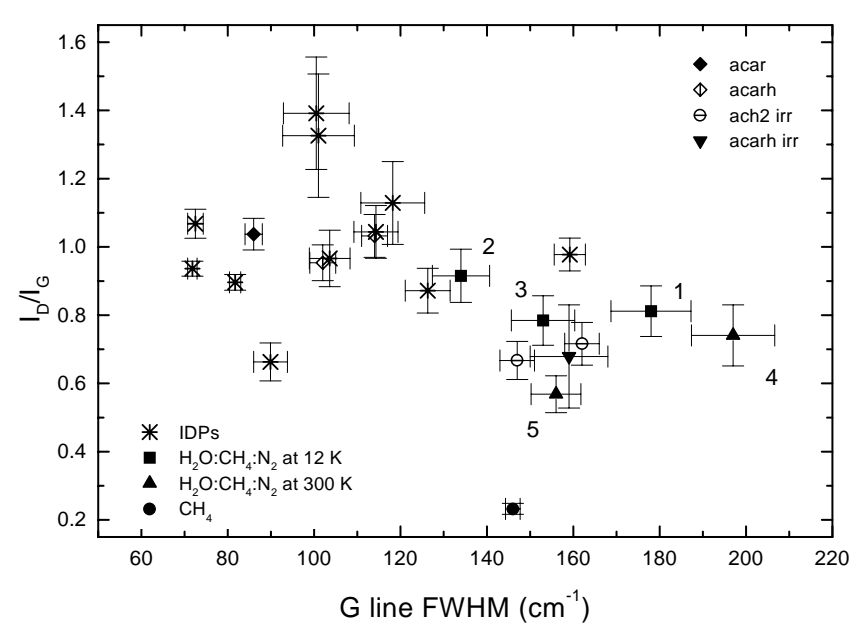

Fig. 10. $I_{\mathrm{D}} / I_{\mathrm{G}}$ ratio versus $\mathrm{G}$ line full width at half maximum $(F W H M)$ for the laboratory samples discussed in this work and 11 IDPs. All the symbols in the graph have the same meaning of the previous figure.

The most ordered IDPs according to the classification made by Wopenka, i.e. those with the narrowest $\mathrm{G}$ and $\mathrm{D}$ lines (viz. Essex, Attila and Tyson), are far from our IPHACs. The IDP which is closer to our irradiated ices is Spray, which has a rather low degree of order. We can conclude that the IDPs here considered have spectral features which are not reproduced by IPHAC samples.

The observed results indicate that the $\mathrm{AC}$ present in the studied IDPs differs from that formed by ion irradiation of frozen gases in the cometary crust. Thus, if the cometary crust is formed after ion irradiation (Strazzulla \& Johnson 1991), these results question the hypothesis that some IDPs could be fragments of the cometary crust. Alternatively, we could imagine that the crust has a different origin or that there is a different mechanism which leads to the amorphous carbon detected in interplanetary grains. These may be, for example, "older" than the crust, in the sense that they could be the sites for the surface ice to condense when the comet forms. In this case, the crust should have a different history and composition from the AC that is embedded in IDPs, suggesting that the crust is very stable.

Some of the considered IDPs might not have a cometary origin. Some of them (namely Essex, Attila, Tyson) probably do have an asteroidal origin, so they could have been processed at high temperature in the inner Solar System. This hypothesis is confirmed by a terrestrial-like deuterium abundance and the presence of crystalline olivine. Depending on the IDPs origin (whether asteroidal or cometary), the interaction with the atmosphere is different. Dust particles from comets generally enter the Earth's atmosphere with more initial energy than that possessed by asteroidal dust particles (see for example Jackson \& Zook 1992) and hence cometary particles of the same size and density, on the average, should be heated more in their deceleration.

This means that IDPs can be differently annealed by the terrestrial atmosphere, which heats them by a considerable amount $\left(T \sim 400-1200^{\circ} \mathrm{C}\right)$ for a few seconds. This heat pulse will alter abundant organic matter in IDPs and, for example, it 
may lead in some cases to vesicle formation, and in very extreme cases to a porous structure, made up of interconnected bubbles (Brownlee et al. 1997, 2002). Anyway, though it is known that thermal annealing increases the degree of order in amorphous carbon (since it tends to remove heteroatoms and structural defects in the amorphous carbon, so that the basic structural carbonaceous units re-arrange, raising the "degree of order") the atmospheric heating alone cannot explain the different spectral characteristic of AC in IDPs. In fact, as Wopenka (1988) points out, Attila and Skywalker, which show nuclear tracks that indicate the same atmospheric heating (below $600{ }^{\circ} \mathrm{C}$ ), exhibit a different AC Raman feature. This could imply differences in chemical composition and/or structure of the precursor material.

All these considerations indicate that the understanding of the evolution of IDPs needs further experimental work and different kinds of samples are to be considered. So other experiments, such as those here presented, which provide an example of comparison between the AC Raman feature of IDPs and of laboratory samples, are needed for further information.

Acknowledgements. We are grateful to F. Spinella for his valuable support in the laboratory. This research has been financially supported by the Italian Ministero dell'Istruzione, dell'Università e della Ricerca (MIUR) and by the Italian Space Agency (ASI).

\section{References}

Baratta, G. A., Arena, M. M., Strazzulla, G., et al. 1996, NIMB, 116, 195

Baratta, G. A., \& Palumbo, M. E. 1998, JOSA, 15, 3076

Baratta, G. A., Leto, G., \& Palumbo, M. E. 2002, A\&A, 384, 343

Brownlee, D. E., Joswiak, D., \& Bradley, J. P. 1997, LPI, 28, 165

Brownlee, D. E., Joswiak, D. J., Kress, M. E., et al. 2002, LPI, 33, 1786

Compagnini, G., Calcagno, L., \& Foti, G. 1992, Phys. Rev. Lett., 69, 444

Compagnini, G., \& Baratta, G. A. 1992, Appl. Phys. Lett., 61, 1796

Dillon, R. O., Wollam, J. A., \& Katkanant, V. 1984, Phys. Rev. B, 29, 3482

Elman, B. S., Dresselhaus, M. S., Dresselhaus, G., Maby, E. W., \& Mazurek, H. 1981, Phys. Rev. B, 24, 1027

Fahey, A., McKeegan, K. D., Sandford, S. A., Wopenka, B., \& Zinner, E. 1985, in Properties and interactions of interplanetary dust, ed. R. H. Giese \& P. Lamy (D. Reidel Pub. Co.), 149

Foti, G., Calcagno, L., Sheng, K. L., \& Strazzulla, G. 1984, Nature, 310,126
Fraundorf, P., Lyons, T., \& Schubert, P. 1982, JGR, 87, A409

Jackson, A. A., \& Zook, H. A. 1992, Icarus, 97, 70

Jenniskens, P., Baratta, G. A., Kouchi, A., et al. 1993, A\&A, 273, 583

Joswiak, D. J., Brownlee, D. E., Pepin, R. O., \& Schlutter, D. J. 2000, LPI, 31, 1500

Kaiser, R. I., \& Roessler, K. 1998, ApJ, 503, 959

Keller, L. P., \& McKay, D. S. 1994, Meteoritics, 29, 480

Macklin, J., Brownlee, D., Chang, S., \& Bunch, T. 1987, in Microbeam analysis, ed. R. H. Geiss, 211

McKeegan, K. D., Sandford, S. A., Walker, R. M., \& Zinner, E. 1984, Meteoritics, 19, 269

Mennella, V., Munoz Caro, G. M., Ruiterkamp, R., et al. 2001, A\&A, 367,355

Mennella, V., Brucato, J. R., Colangeli, L., \& Palumbo, P. 2002, ApJ, 569,531

Mennella, V., Baratta, G. A., Esposito, A., Ferini, G., \& Pendleton, Y. J. 2003, ApJ, 587, 727

Moore, M. H., Donn, B., Khanna, R., \& A'Hearn, M. F. 1983, Icarus, 54,388

Moore, M. H., \& Hudson, R. H. 2003, Icarus, 161, 486

Musumeci, P., Calcagno, L., Makhtari, A., et al. 2000, NIMPB, 166, 404

Raynal, P. I., Quirico, E., Borg, J., \& d'Hendecourt, L. 2001, Lunar and Planetary Science XXXII, abstract no. 1341

Robertson, J. 1986, Adv. Phys., 35, 317

Sandford, S. A., \& Walker, R. M. 1985, ApJ, 291, 838

Soderblom, L. A., Becker, T. L., Bennett, G., et al. 2002, Science, 296, 1087

Strazzulla, G., \& Baratta, G. A. 1992, A\&A, 266, 434

Strazzulla, G., \& Johnson, R. E. 1991, in Comets in the Post-Halley Era, ed. R. Newburn Jr., M. Neugebauer, \& J. Rahe (Dordrecht: Kluwer), 243

Strazzulla, G., Calcagno, L., \& Foti, G. 1983, MNRAS, 204, 59

Strazzulla, G., Leto, G., Baratta, G. A., \& Spinella, F. 1991, JGR, 96, 17547

Strazzulla, G., Baratta, G. A., \& Palumbo, M. E. 2001, Spectrochim. Acta, 57, 825

Thomas, K. L., Blanford, G. E., Keller, L. P., Klock, W., \& McKay, D. S. 1993, GeCo Acta, 57, 1551

Thomas, K. L., Keller, L. P., Blanford, G. E., McKay, D. S. 1994, AIP Conf. Proc., 310, 165

Thomas, K. L., Keller, L. P., \& McKay, D. S. 1995, Meteoritics, 30, 587

Tuinstra, F., \& Koenigh, J. L. 1970, J. Chem. Phys., 53, 1126

Vidano, R. P. 1980, Ph.D. Thesis, University of Washington

Vidano, R. P., \& Fishbach, D. B. 1981, Extended Abstract of the 15th Biennal Conf. on Carbon, ed. F. L. Vogel, \& W. C. Foresman, 468

Wang, A., Jolliff, B. L., Haskin, L. A., \& Kuebler, K. E. 2000, LPS, 31,1875

Wopenka, B. 1988, Earth Planet. Sci. Lett., 88, 221 